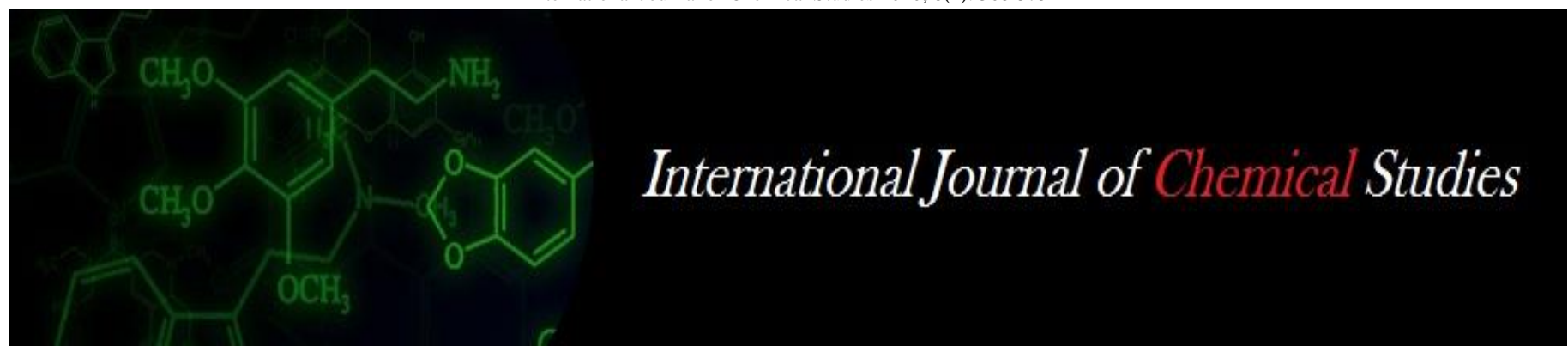

P-ISSN: 2349-8528

E-ISSN: 2321-4902

IJCS 2020; 8(1): 368-375

(C) 2020 IJCS

Received: 19-11-2019

Accepted: 21-12-2019

\section{R Divya Madhuri}

Regional Agricultural Research Station, Nandyal, Kurnool

(Dist.), Andhra Pradesh, India

\section{Jayalakshmi}

Regional Agricultural Research

Station, Nandyal, Kurnool

(Dist.), Andhra Pradesh, India
Corresponding Author:

R Divya Madhuri

Regional Agricultural Research

Station, Nandyal, Kurnool

(Dist.), Andhra Pradesh, India

\section{Identification of high yielding mechanical harvestable chickpea (Cicer arietinum $\mathrm{L}$.) genotypes under rainfed and irrigated conditions}

\author{
R Divya Madhuri and V Jayalakshmi
}

DOI: https://doi.org/10.22271/chemi.2020.v8.i1e.8274

\begin{abstract}
Thirty chickpea genotypes were evaluated at Regional Agricultural Research Station, Nandyal, Andhra Pradesh, India during rabi, 2018- 2019 with three replications both under rainfed and irrigated conditions utilising yield and mechanical harvestable traits. The analysis of variance revealed highly significant differences among the genotypes for all the traits under study under rainfed as well as irrigated conditions. This study was conducted to determine high yielding mechanical harvestable genotypes in rainfed and irrigated conditions. NBeG 776, NBeG 779 and NBeG 868 are suitable under both rainfed and irrigated conditions with significantly higher yields over their respective means. ICCV 181606, MH 13 and MH 14 are suitable exclusively for rainfed condition with significantly superior yields over the mean. ICCV 181607 that produced high yield along with machine harvestable traits should be deployed in breeding programmes planned to improve yield as well as machine harvestablity.
\end{abstract}

Keywords: Chickpea, yield, rainfed and irrigated

\section{Introduction}

India is the world's largest producer of chickpea with annual production of 9.07 million tonnes from an area of 9.54 million ha with productivity of $951.4 \mathrm{~kg} \mathrm{ha}^{-1}$ (FAO STAT, 2019) ${ }^{[5]}$. In Southern India, Andhra Pradesh has witnessed dramatic increase in chickpea area from 71,000 ha in 1992-93 to 5.86 lakh ha during 2013-14 registering the highest productivity of $1449 \mathrm{~kg}$ $\mathrm{ha}^{-1}$ (AICRP on Chickpea, Annual report, 2017-18). Introduction and large scale adoption of short duration, wilt resistant varieties such as 'JG 11', 'JAKI 9218' and 'KAK 2' and mechanization of farming operations have contributed for realisation of higher productivity levels. However, these existing varieties have semi spreading growth habit and are not suitable for machine harvesting. Moreover, due to short growing season, the growth of the crop in Andhra Pradesh is less and existing popular varieties attain a plant height of $35-40 \mathrm{~cm}$ during warm and short growing season. Therefore, chickpea crop is largely harvested manually and partial mechanization i.e., threshing is done by combine harvester. Complete mechanization (machine harvesting) is cost effective and quicker, reducing the risk of ripened crop's exposure to untimely rain or other extreme weather conditions. Tall chickpea cultivars with 30 to $40 \%$ more height than the existing cultivars and semi-erect to erect growth habit with branching starting from $25-30 \mathrm{~cm}$ and above are suitable to mechanical harvesting. There is pressing need to breed chickpea varieties amenable to machine harvesting, with yield potential equal to or higher than existing popular cultivars. Breeding programmes were initiated through collaborative programmes of ICRISAT, ICAR Institutes and State Agricultural Universities to develop tall and semi-erect chickpea cultivars suitable to machine harvesting. Location specific breeding programmes at Regional Agricultural Research Station, Nandyal of Acharya N G Ranga Agricultural University in Andhra Pradesh state of India have led to development of a chickpea cultivar Dheera (NBeG 47) which is suited to mechanical harvesting (Jayalakshmi et al., 2017). Mannur et al. (2013) ${ }^{[7,12]}$ developed a new variety of chickpea GBM-2 at ARS, Gulbarga, Karnataka which is 1.5 to 2 times taller than the traditional varieties.

In chickpea greater proportion of photosynthetic assimilates are allocated to pods and seeds when crop experiences moisture stress after flowering or when it was raised completely 
without irrigation (Deshmukh et al., 2004) ${ }^{[4]}$. There is a need to evaluate existing cultivars under cultivation and advance breeding lines developed in the location specific research programmes of the region for drought stress tolerance under drought stress (rainfed) as well as non-stress (irrigated) conditions to understand drought management mechanism of genotypes and to quantify the yield losses due to these stresses in rainfed conditions. Simultaneously, this evaluation enables to identify chickpea genotypes which can perform well during rainfed situations with plasticity to respond to availability of soil moisture during good rainfall years.

\section{Materials and Methods}

The investigation was carried out during rabi 2018-19 at Regional Agricultural Research Station, Nandyal, situated at $15^{\circ} 29^{\prime}$ North latitude and $78^{0} 29^{\prime}$ East longitude at an altitude of $211.76 \mathrm{~m}$ above mean sea level. The research station comes under scarce rainfall zone of Andhra Pradesh. The experimental material comprised of 25 desi chickpea genotypes and five checks viz., NBeG 47, NBeG 49, JG 11, GBM 2 and HC 5 which were sown on $24^{\text {th }}$ October rabi, 2018 in a Randomized Block Design (RBD) with three replications under both rainfed and irrigated situations. Since very meagre rainfall was received during rabi season, a pre sowing irrigation was given to take up the sowing of experiments. In rainfed condition, genotypes were grown on receding soil moisture where as in irrigated condition two supplemental irrigations were given at 35 and 55 days after sowing through sprinklers. Each genotype was sown in two rows in a plot of $3 \mathrm{~m}$ row length at spacing of $30 \mathrm{~cm}$ between rows and $10 \mathrm{~cm}$ between plants within the row.

\section{Results and Discussion \\ Analysis of variance}

The analysis of variance was performed for each character separately under rainfed and irrigated conditions and the total variation was partitioned into different sources of variation. The mean squares due to treatments were significant for all traits under rainfed and irrigated conditions. This indicated genetic variation among genotypes of the present investigation for traits under study. The results are presented in the Table 1.

The mean performance of thirty chickpea genotypes evaluated under rainfed and irrigated conditions is presented in Table 2.

Table 1: Analysis of variance for 14 characters in 30 chickpea genotypes under rainfed and irrigated condition during rabi 2018-19

\begin{tabular}{|c|c|c|c|c|c|c|c|}
\hline \multirow{4}{*}{ S. No. } & \multirow{4}{*}{ Character } & \multicolumn{6}{|c|}{ Mean squares } \\
\hline & & \multirow{2}{*}{\multicolumn{2}{|c|}{$\begin{array}{c}\text { Replications } \\
\text { (df : } 2)\end{array}$}} & \multirow{2}{*}{\multicolumn{2}{|c|}{$\begin{array}{c}\text { Treatments } \\
\text { (df :29) }\end{array}$}} & \multirow{2}{*}{\multicolumn{2}{|c|}{$\begin{array}{c}\text { Error } \\
(d f: 58)\end{array}$}} \\
\hline & & & & & & & \\
\hline & & Rainfed & Irrigated & Rainfed & Irrigated & Rainfed & Irrigated \\
\hline 1 & Angle of the primary branch $\left(^{\circ}\right)$ & 0.57 & 2.55 & $25.22 * *$ & $26.12 * *$ & 2.10 & 1.57 \\
\hline 2 & Height of the first pod $(\mathrm{cm})$ & 1.72 & $10.62 * *$ & $52.88 * *$ & $37.94 * *$ & 1.36 & 1.42 \\
\hline 3 & Days to $50 \%$ flowering & 1.64 & 8.84 & $140.18 * *$ & $159.99 * *$ & 2.20 & 4.15 \\
\hline 4 & Days to maturity & 0.68 & 0.63 & $84.50 * *$ & $82.40 * *$ & 1.93 & 0.54 \\
\hline 5 & Duration of reproductive phase (days) & 2.01 & 2.03 & $25.63 * *$ & $38.84 * *$ & 4.03 & 5.63 \\
\hline 6 & Plant height $(\mathrm{cm})$ & 1.88 & 1.12 & $48.33 * *$ & $52.75 * *$ & 1.13 & 1.93 \\
\hline 7 & No. of primary branches plant ${ }^{-1}$ & 0.03 & 0.11 & $0.73 * *$ & $0.47 * *$ & 0.07 & 0.09 \\
\hline 8 & No. of secondary branches plant ${ }^{-1}$ & $2.53 *$ & 0.69 & $15.23 * *$ & $3.73 * *$ & 0.79 & 0.71 \\
\hline 9 & No. of pods plant ${ }^{-1}$ & 1.40 & 8.08 & $109.24 * *$ & $139.42 * *$ & 3.50 & 35.40 \\
\hline 10 & Biological yield $\operatorname{plot}^{-1}(2.8 \mathrm{~m} \times 0.6 \mathrm{~m})(\mathrm{g})$ & 994.68 & 3179.54 & $25830.96^{* *}$ & $23266.00^{* *}$ & 2209.38 & 5383.05 \\
\hline 11 & Harvest index $(\%)$ & 21.51 & 3.79 & $331.71 * *$ & $168.02 * *$ & 9.03 & 5.59 \\
\hline 12 & 100 seed weight $(\mathrm{g})$ & 0.63 & 0.90 & $64.38 * *$ & $55.16 * *$ & 0.62 & 0.62 \\
\hline 13 & Protein $(\%)$ & 4.15 & 1.76 & $8.51 * *$ & $25.03 * *$ & 3.16 & 1.18 \\
\hline 14 & Seed yield plot $^{-1}(2.8 \mathrm{~m} \times 0.6 \mathrm{~m})(\mathrm{g})$ & 520.81 & 870.18 & $15793.39 * *$ & $15145.75^{* *}$ & 512.82 & 1537.97 \\
\hline
\end{tabular}

* Significant at $\mathrm{P} \leq 0.05, * *$ Significant at $\mathrm{P} \leq 0.01$

Table 2: Per se performance of 30 chickpea genotypes for 14 characters under rainfed and irrigated condition during rabi 2018-19

\begin{tabular}{|c|c|c|c|c|c|c|c|c|c|}
\hline S. No. & Genotypes & Angle of th & branch $\left(^{\circ}\right)$ & Height of & $\operatorname{pod}(\mathrm{cm})$ & Days to 5 & lowering & Days to & aturity \\
\hline & & $\mathrm{RF}$ & IR & $\mathrm{RF}$ & IR & $\mathrm{RF}$ & IR & RF & IR \\
\hline 1 & NBeG 776 & 73.8 & 74.9 & 20.6 & 22.3 & $34.3 * *$ & $35.3 * *$ & $81.0 * *$ & $82.3 * *$ \\
\hline 2 & NBeG 779 & 75.5 & 77.5 & 20.1 & 21.7 & $35.3 * *$ & $35.3 * *$ & $81.3 * *$ & $81.7 * *$ \\
\hline 3 & NBeG 780 & $80.5^{* *}$ & $79.7 * *$ & 26.1 & 25.7 & $36.3 * *$ & $36.7 * *$ & $82.0 * *$ & $83.3 * *$ \\
\hline 4 & NBeG 865 & 73.7 & 71.4 & $27.7 *$ & 28.1 & 49.3 & 57.3 & 96.0 & 98.7 \\
\hline 5 & NBeG 868 & 71.2 & 73.7 & 22.2 & 20.5 & $36.3 * *$ & $37.3 * *$ & $82.3 * *$ & $84.0 * *$ \\
\hline 6 & PG 08108 & 72.9 & 70.9 & 25.3 & 23.7 & 52.0 & 52.0 & 96.3 & 96.0 \\
\hline 7 & ICCV 08102 & 73.7 & 72.9 & 22.9 & 23.1 & 53.7 & 51.7 & 96.3 & 97.0 \\
\hline 8 & ICCV 181602 & $77.5^{*}$ & $78.3^{*}$ & $28.3 * *$ & $29.7 * *$ & 52.7 & 52.3 & 96.7 & 98.0 \\
\hline 9 & ICCV 181606 & 74.2 & $78.3^{*}$ & 23.9 & 24.9 & $40.7 * *$ & $41.0 * *$ & 87.3 & $88.0 * *$ \\
\hline 10 & ICCV 181607 & 75.5 & 77.1 & $28.7 * *$ & $29.3 * *$ & 50.3 & 51.7 & 85.7 & $86.0^{* * *}$ \\
\hline 11 & ICCV 181608 & $78.8 * *$ & $79.3 * *$ & $30.1 * *$ & $29.3 * *$ & 50.3 & 49.3 & 89.7 & 89.0 \\
\hline 12 & ICCV 181610 & 73.9 & $78.7 * *$ & $28.7 * *$ & $29.2 * *$ & 51.7 & 52.7 & $86.7 *$ & $87.3 * *$ \\
\hline 13 & ICCV 181612 & 69.2 & 77.5 & $30.7 * *$ & 27.4 & 50.0 & 49.7 & $86.0 * *$ & $87.0 * *$ \\
\hline 14 & ICCV 181664 & 73.1 & 77.8 & $35.4 * *$ & $34.9 * *$ & 50.0 & 50.3 & 96.7 & 96.7 \\
\hline 15 & ICCV 181667 & 71.3 & 74.5 & $27.8^{*}$ & $32.5 * *$ & 50.0 & 46.3 & 92.3 & 95.0 \\
\hline 16 & MH 1 & 75.3 & 74.8 & 25.5 & 24.8 & 44.0 & 47.3 & 89.3 & 89.3 \\
\hline 17 & MH 4 & 75.9 & 73.7 & 20.5 & 25.7 & 45.3 & 49.7 & 90.3 & 89.0 \\
\hline 18 & MH 5 & $77.5^{*}$ & 74.1 & $30.1 * *$ & 27.8 & 53.0 & 51.7 & 93.7 & 94.0 \\
\hline 19 & MH 11 & 76.4 & $83.2 * *$ & 25.2 & $28.6^{*}$ & 45.0 & 46.7 & $85.3 * *$ & $87.7 * *$ \\
\hline 20 & MH 12 & 75.0 & 74.7 & 23.5 & 25.6 & $40.7 * *$ & 45.7 & 88.0 & 90.3 \\
\hline
\end{tabular}




\begin{tabular}{|c|c|c|c|c|c|c|c|c|c|}
\hline 21 & MH 13 & 74.5 & 75.1 & 18.9 & 24.3 & 45.3 & 44.3 & 90.7 & 89.7 \\
\hline 22 & MH 14 & 74.9 & 75.9 & 18.4 & 22.5 & 44.0 & $40.3 * *$ & 91.0 & 90.3 \\
\hline 23 & MH 15 & 75.5 & 73.9 & 22.7 & 26.6 & 44.7 & $42.0 * *$ & 88.3 & $86.3 * *$ \\
\hline 24 & MH 21 & 71.1 & 74.1 & $28.7 * *$ & 25.3 & 53.3 & 55.0 & 92.7 & 95.3 \\
\hline 25 & MH 22 & 72.7 & 75.5 & 26.9 & 27.1 & 52.0 & 50.0 & 94.0 & 93.3 \\
\hline 26 & $\mathrm{NBeG} 47$ & $78.5 * *$ & $80.9 * *$ & 25.1 & $32.3 * *$ & $40.3 * *$ & $40.7 * *$ & $85.3 * *$ & $88.7 *$ \\
\hline 27 & NBeG 49 & 73.6 & 72.3 & 20.1 & 20.7 & $35.0 * *$ & $35.7 * *$ & $83.7 * *$ & $84.3 * *$ \\
\hline 28 & JG 11 & 69.1 & 71.5 & 19.0 & 21.7 & $35.3^{* *}$ & $34.3 * *$ & $81.3^{* *}$ & $82.3^{* *}$ \\
\hline 29 & GBM 2 & 76.1 & 74.7 & $29.0 * *$ & 25.2 & 58.0 & 58.7 & 96.3 & 96.7 \\
\hline 30 & HC 5 & $81.1 * *$ & $78.0 * *$ & $28.2 * *$ & 27.8 & 51.7 & 58.7 & 96.3 & 96.7 \\
\hline \multicolumn{2}{|c|}{ General Mean } & 74.7 & 75.8 & 25.3 & 26.3 & 46.0 & 46.7 & 89.4 & 90.1 \\
\hline \multicolumn{2}{|c|}{ CV $(\%)$} & 1.9 & 1.6 & 4.6 & 4.5 & 3.2 & 4.4 & 1.6 & 0.8 \\
\hline \multicolumn{2}{|c|}{$\mathrm{SE}(\mathrm{m})$} & 0.8 & 0.7 & 0.7 & 0.7 & 0.9 & 1.2 & 0.8 & 0.4 \\
\hline \multicolumn{2}{|c|}{$\mathrm{CD}(\mathrm{P} \leq 0.05)$} & 2.4 & 2.0 & 1.9 & 1.9 & 2.4 & 3.3 & 2.3 & 1.2 \\
\hline \multicolumn{2}{|c|}{$\mathrm{CD}(\mathrm{P} \leq 0.01)$} & 3.1 & 2.7 & 2.5 & 2.6 & 3.2 & 4.4 & 3.0 & 1.6 \\
\hline
\end{tabular}

\section{S. No. Genotypes Duration of reproductive phase (days) Plant height (cm) No. of primary branches plant ${ }^{-1}$}

No. of secondary branches

\begin{tabular}{|c|c|c|c|c|c|c|c|c|c|}
\hline \multirow[t]{2}{*}{ S. No. } & \multirow[t]{2}{*}{ Genotypes } & \multicolumn{2}{|c|}{ Duration of reproductive pnase (aays } & \multicolumn{2}{|c|}{ Prant neignt (cm) } & \multicolumn{2}{|c|}{ No. or primary drancines plant } & \multicolumn{2}{|c|}{ plant $^{-1}$} \\
\hline & & RF & IR & $\mathrm{RF}$ & IR & $\mathrm{RF}$ & IR & $\mathrm{RF}$ & IR \\
\hline 1 & NBeG 776 & 50.3 & 51.3 & 36.5 & 40.1 & 2.1 & 2.1 & $11.0^{* *}$ & 8.3 \\
\hline 2 & NBeG 779 & 48.0 & 49.0 & 34.9 & 36.1 & 1.4 & 2.1 & 6.3 & 7.8 \\
\hline 3 & NBeG 780 & 49.3 & 49.0 & $41.6^{* *}$ & 42.1 & 2.2 & 2.3 & $10.9 * *$ & 8.3 \\
\hline 4 & NBeG 865 & 48.3 & 53.0 & 35.3 & 41.4 & $3.4 * *$ & $3.3^{*}$ & 6.9 & 8.2 \\
\hline 5 & NBeG 868 & 46.7 & 48.7 & 33.3 & 35.9 & 2.2 & 2.3 & $13.4 * *$ & $9.5^{*}$ \\
\hline 6 & PG 08108 & 51.7 & 52.3 & 36.9 & 37.3 & 2.7 & 2.7 & 7.9 & 7.3 \\
\hline 7 & ICCV 08102 & 53.0 & $58.3 * *$ & 39.1 & 36.5 & 2.6 & 2.4 & 6.7 & 6.5 \\
\hline 8 & ICCV 181602 & 44.0 & 45.7 & $43.0^{* * *}$ & $45.7 * *$ & $3.1 *$ & 3.1 & 8.5 & 7.9 \\
\hline 9 & ICCV 181606 & $54.3 *$ & $56.3 * *$ & 37.2 & 39.7 & 2.5 & 2.9 & 7.1 & 6.9 \\
\hline 10 & ICCV 181607 & 51.7 & 53.3 & $43.1 * *$ & $44.0 * *$ & $3.0^{*}$ & 3.1 & 7.0 & 7.0 \\
\hline 11 & ICCV 181608 & 52.0 & $56.0 *$ & $44.8 * *$ & $45.7 * *$ & 2.5 & 2.8 & 7.5 & 7.8 \\
\hline 12 & ICCV 181610 & 53.0 & $55.0 *$ & $43.3 * *$ & $45.8^{* *}$ & 2.3 & 2.6 & 6.9 & 6.6 \\
\hline 13 & ICCV 181612 & 48.0 & 50.7 & $41.0^{*}$ & 40.2 & 2.9 & 3.0 & 6.9 & 8.5 \\
\hline 14 & ICCV 181664 & 45.0 & 46.7 & $49.5 * *$ & $50.3 * *$ & $3.3 * *$ & $3.3^{*}$ & 7.9 & 8.1 \\
\hline 15 & ICCV 181667 & 51.3 & 51.3 & $45.1 * *$ & 41.9 & $3.1 *$ & $3.3 *$ & 8.8 & 8.8 \\
\hline 16 & MH 1 & 51.3 & 54.3 & 35.8 & 37.2 & 2.4 & 2.9 & 7.1 & 8.1 \\
\hline 17 & MH 4 & 49.0 & 47.0 & 34.2 & 37.2 & $3.3^{* *}$ & 2.8 & 8.2 & 7.0 \\
\hline 18 & MH 5 & 50.0 & 51.3 & 39.9 & 40.7 & 2.5 & 2.7 & 6.5 & 7.7 \\
\hline 19 & MH 11 & 49.7 & 44.7 & 37.6 & $43.7 * *$ & 2.3 & 2.4 & 8.1 & 6.8 \\
\hline 20 & MH 12 & 51.7 & 47.3 & 33.8 & 40.9 & 1.9 & 2.7 & 4.2 & 7.1 \\
\hline 21 & MH 13 & $55.0 *$ & 52.0 & 36.1 & 35.7 & 2.9 & 3.1 & 6.3 & 8.9 \\
\hline 22 & MH 14 & 52.3 & 47.7 & 34.4 & 35.9 & 2.9 & 2.7 & 8.8 & 7.6 \\
\hline 23 & MH 15 & 50.0 & 44.3 & 37.7 & 40.3 & 2.1 & 2.3 & 4.9 & 8.0 \\
\hline 24 & MH 21 & $58.0 * *$ & 53.3 & 38.9 & 34.2 & 2.6 & 2.6 & 6.1 & 6.9 \\
\hline 25 & MH 22 & 53.0 & $55.3 *$ & 40.1 & 41.5 & $3.1^{*}$ & 2.5 & 7.4 & 7.7 \\
\hline 26 & NBeG 47 & 51.0 & 48.7 & $42.3 * *$ & $46.0 * *$ & 1.7 & 1.8 & 5.4 & 7.3 \\
\hline 27 & NBeG 49 & 49.0 & 53.3 & 37.7 & 38.6 & 2.0 & 2.4 & $12.9 * *$ & $11.9 * *$ \\
\hline 28 & JG 11 & 48.3 & 50.3 & 33.7 & 30.9 & 2.3 & 2.3 & $11.9 * *$ & $9.9 * *$ \\
\hline 29 & GBM 2 & 53.3 & 53.7 & $42.0^{* *}$ & 39.4 & 2.4 & 2.9 & 6.1 & 8.8 \\
\hline 30 & HC 5 & 52.3 & 51.0 & 40.5 & 42.4 & 2.5 & 3.2 & 5.5 & 7.9 \\
\hline \multicolumn{2}{|c|}{ General Mean } & 50.7 & 51.0 & 39.0 & 40.2 & 2.5 & 2.7 & 7.8 & 8.0 \\
\hline \multicolumn{2}{|c|}{$\mathrm{CV}(\%)$} & 4.0 & 4.6 & 2.7 & 3.5 & 10.6 & 11.3 & 11.4 & 10.6 \\
\hline \multicolumn{2}{|r|}{$\mathrm{SE}(\mathrm{m})$} & 1.2 & 1.4 & 0.6 & 0.8 & 0.2 & 0.2 & 0.5 & 0.5 \\
\hline \multicolumn{2}{|c|}{$\mathrm{CD}(\mathrm{P} \leq 0.05)$} & 3.3 & 3.9 & 1.7 & 2.3 & 0.4 & 0.5 & 1.5 & 1.4 \\
\hline \multicolumn{2}{|c|}{$\mathrm{CD}(\mathrm{P} \leq 0.01)$} & 4.4 & 5.2 & 2.3 & 3.0 & 0.6 & 0.7 & 1.9 & 1.8 \\
\hline
\end{tabular}

\begin{tabular}{|l|c|c|c|c|c|}
\hline S. No. & Genotypes & No. of pods plant & \\
\hline & & Biological yield plot $^{-1}(\mathrm{~g})\left(2.8 \mathbf{~ m}^{*} \mathbf{0 . 6 m}\right)$ & Harvest index $(\%)$ & 100 seed weight $(\mathrm{g})$ \\
\hline
\end{tabular}

\begin{tabular}{|c|c|c|c|c|c|c|c|c|c|}
\hline 3. No. & Genotypes & No. of $\mathrm{p}$ & plant & Biological y & $.8 \mathrm{~m}(0.6 \mathrm{~m})$ & Harvest & $\operatorname{dex}(\%)$ & 100 see & eight (g \\
\hline & & $\mathrm{RF}$ & IR & RF & IR & RF & IR & RF & IR \\
\hline 1 & NBeG 776 & 25.9 & 28.0 & 540.0 & 706.7 & $59.2 * *$ & $53.9 * *$ & $29.3^{* *}$ & $28.3^{* *}$ \\
\hline 2 & NBeG 779 & 29.7 & 25.5 & 556.7 & 716.7 & $54.9 * *$ & $54.6 * *$ & $29.7 * *$ & $29.3 * *$ \\
\hline 3 & NBeG 780 & 29.2 & 19.1 & 460.0 & 523.3 & $52.5 * *$ & 43.3 & $31.7 * *$ & $31.0 * *$ \\
\hline 4 & NBeG 865 & 24.2 & 34.0 & 573.3 & $776.7 *$ & 31.7 & 48.9 & $32.3 * *$ & $33.7 * *$ \\
\hline 5 & NBeG 868 & 29.9 & 28.1 & 613.3 & $756.7^{*}$ & $59.0 * *$ & $56.4 * *$ & 24.7 & 26.3 \\
\hline 6 & PG 08108 & 26.2 & 32.5 & 536.7 & 566.7 & 39.8 & 47.2 & 20.3 & 23.7 \\
\hline 7 & ICCV 08102 & 26.7 & 30.7 & 528.3 & 673.3 & 26.7 & $51.7 *$ & 25.0 & 25.3 \\
\hline 8 & ICCV 181602 & 18.1 & 17.9 & 513.3 & 523.3 & 30.5 & 31.2 & $30.7 * *$ & $31.7 * *$ \\
\hline 9 & ICCV 181606 & $37.1 * *$ & 40.8 & 543.3 & 650.0 & $56.0 * *$ & 49.8 & 24.7 & 25.7 \\
\hline 10 & ICCV 181607 & $38.5^{* *}$ & 33.8 & 580.0 & 673.3 & $51.7 * *$ & 42.3 & $32.0^{* *}$ & $32.7 * *$ \\
\hline 11 & ICCV 181608 & $34.2 * *$ & 30.4 & 520.0 & 620.0 & 44.9 & 42.5 & 25.0 & 25.3 \\
\hline 12 & ICCV 181610 & 24.1 & 26.3 & 600.0 & 623.3 & 36.7 & 37.0 & 23.7 & 23.0 \\
\hline 13 & ICCV 181612 & 28.2 & 33.5 & 533.3 & 610.0 & 43.8 & 40.6 & 20.3 & 20.3 \\
\hline
\end{tabular}




\begin{tabular}{|c|c|c|c|c|c|c|c|c|c|}
\hline 14 & ICCV 181664 & 25.2 & 21.1 & 496.7 & 527.7 & 28.0 & 26.9 & $27.3 * *$ & $27.7 * *$ \\
\hline 15 & ICCV 181667 & 27.8 & 29.0 & 510.0 & 536.7 & 35.3 & 36.6 & 20.3 & 20.3 \\
\hline 16 & MH 1 & 25.7 & 28.9 & $736.7 * *$ & 603.3 & 42.8 & 40.4 & 21.7 & 21.7 \\
\hline 17 & $\mathrm{MH} 4$ & $33.4 * *$ & 30.8 & 540.0 & $850.0 * *$ & 37.6 & 45.1 & 22.3 & 22.0 \\
\hline 18 & MH 5 & 30.4 & 39.5 & 540.0 & 663.3 & 26.1 & 48.2 & 16.0 & 17.7 \\
\hline 19 & MH 11 & 20.8 & 23.1 & 416.7 & 700.0 & 44.9 & 50.5 & $26.7 * *$ & $29.0 * *$ \\
\hline 20 & MH 12 & 16.0 & 38.1 & 468.0 & 660.0 & 48.3 & $52.1 * *$ & 19.3 & 19.7 \\
\hline 21 & MH 13 & $35.4 * *$ & 39.5 & $820.0 * *$ & $790.0^{*}$ & 38.6 & 40.6 & 19.3 & 21.7 \\
\hline 22 & MH 14 & $38.9 * *$ & 31.5 & $740.0 * *$ & 636.7 & $54.6 * *$ & $55.6 * *$ & $28.0 * *$ & 26.7 \\
\hline 23 & MH 15 & 26.3 & $47.9 * *$ & 519.3 & $793.3^{*}$ & 40.8 & 48.7 & 21.7 & 22.0 \\
\hline 24 & MH 21 & 21.2 & 32.2 & 480.0 & 695.0 & 37.9 & 49.0 & 21.0 & 24.0 \\
\hline 25 & MH 22 & 27.1 & $42.5 *$ & 430.0 & 620.0 & 34.8 & $52.4 * *$ & 21.7 & 23.3 \\
\hline 26 & NBeG 47 & 20.3 & 27.7 & 388.3 & 490.0 & 47.7 & $54.0 * *$ & $31.0 * *$ & $29.0 * *$ \\
\hline 27 & NBeG 49 & $31.7 *$ & 32.8 & 570.3 & 700.0 & $59.6 * *$ & $53.3 * *$ & $31.3 * *$ & $31.0 * *$ \\
\hline 28 & JG 11 & $34.3 * *$ & 32.5 & 493.3 & 616.7 & $59.8 * *$ & $53.6 * *$ & 24.7 & $27.0 * *$ \\
\hline 29 & GBM 2 & 21.6 & 30.9 & 584.3 & 630.0 & 41.8 & $54.2 * *$ & 25.7 & 23.7 \\
\hline 30 & HC 5 & 20.1 & 38.2 & 463.3 & 686.7 & 34.4 & 43.2 & 18.7 & 19.3 \\
\hline \multicolumn{2}{|c|}{ General Mean } & 27.6 & 31.6 & 543.2 & 654.0 & 43.6 & 46.8 & 24.9 & 25.4 \\
\hline \multicolumn{2}{|c|}{$\mathrm{CV}(\%)$} & 6.8 & 18.9 & 8.7 & 11.2 & 6.9 & 5.1 & 3.2 & 3.1 \\
\hline \multicolumn{2}{|c|}{$\mathrm{SE}(\mathrm{m})$} & 1.1 & 3.4 & 27.1 & 42.4 & 1.7 & 1.4 & 0.5 & 0.5 \\
\hline \multicolumn{2}{|c|}{$\mathrm{CD}(\mathrm{P} \leq 0.05)$} & 3.1 & 9.7 & 76.8 & 119.9 & 4.9 & 3.9 & 1.3 & 1.3 \\
\hline \multicolumn{2}{|c|}{$\mathrm{CD}(\mathrm{P} \leq 0.01)$} & 4.1 & 12.9 & 102.2 & 159.5 & 6.5 & 5.1 & 1.7 & 1.7 \\
\hline
\end{tabular}

\begin{tabular}{|c|c|c|c|c|c|}
\hline \multirow[t]{2}{*}{ S. No. } & \multirow[t]{2}{*}{ Genotypes } & \multicolumn{2}{|c|}{ Protein $(\%)$} & \multicolumn{2}{|c|}{ Seed yield plot $^{-1}(\mathrm{~g})(2.8 \mathrm{~m} * 0.6 \mathrm{~m})$} \\
\hline & & RF & IR & RF & IR \\
\hline 1 & NBeG 776 & 17.9 & 20.0 & $318.3 * *$ & $383.7 *$ \\
\hline 2 & NBeG 779 & 17.5 & 15.2 & $305.0 * *$ & $391.3 *$ \\
\hline 3 & NBeG 780 & 17.7 & 18.9 & 241.3 & 226.7 \\
\hline 4 & NBeG 865 & 20.5 & $24.2 * *$ & 180.3 & $380.0^{*}$ \\
\hline 5 & NBeG 868 & 17.0 & 16.6 & $361.7 * *$ & $426.7 * *$ \\
\hline 6 & PG 08108 & 16.8 & 17.5 & 213.3 & 266.7 \\
\hline 7 & ICCV 08102 & 16.5 & $21.5^{* *}$ & 141.3 & 346.7 \\
\hline 8 & ICCV 181602 & 16.8 & 14.7 & 156.7 & 163.3 \\
\hline 9 & ICCV 181606 & 18.9 & 18.7 & $303.3 * *$ & 323.3 \\
\hline 10 & ICCV 181607 & 18.2 & 18.7 & $300.0 * *$ & 284.7 \\
\hline 11 & ICCV 181608 & 18.2 & $24.5^{* *}$ & 233.3 & 263.3 \\
\hline 12 & ICCV 181610 & 20.0 & 20.1 & 220.0 & 230.0 \\
\hline 13 & ICCV 181612 & $22.2 * *$ & 18.9 & 233.3 & 247.3 \\
\hline 14 & ICCV 181664 & 20.0 & 20.5 & 139.0 & 141.7 \\
\hline 15 & ICCV 181667 & 17.3 & 18.6 & 179.3 & 196.7 \\
\hline 16 & MH 1 & 18.9 & $21.4^{* *}$ & $315.0 * *$ & 243.3 \\
\hline 17 & $\mathrm{MH} 4$ & 17.6 & $22.4^{* *}$ & 203.3 & $383.3^{*}$ \\
\hline 18 & MH 5 & 15.7 & 15.1 & 141.7 & 320.0 \\
\hline 19 & MH 11 & 18.2 & $23.5 * *$ & 217.3 & 353.3 \\
\hline 20 & MH 12 & 16.3 & 14.5 & 227.0 & 343.0 \\
\hline 21 & MH 13 & 19.4 & 14.7 & $316.7 * *$ & 322.7 \\
\hline 22 & MH 14 & 18.9 & 18.9 & $403.3 * *$ & 353.3 \\
\hline 23 & MH 15 & 18.6 & 18.2 & 211.7 & $386.7 *$ \\
\hline 24 & MH 21 & 14.7 & 16.6 & 181.7 & 341.7 \\
\hline 25 & MH 22 & 15.1 & 16.8 & 150.0 & 325.0 \\
\hline 26 & NBeG 47 & 18.6 & 19.4 & 185.3 & 264.0 \\
\hline 27 & NBeG 49 & 20.6 & 19.4 & $340.0 * *$ & $373.3^{*}$ \\
\hline 28 & JG 11 & 18.4 & 18.6 & $295.3 * *$ & 330.0 \\
\hline 29 & GBM 2 & 20.0 & $24.2^{* *}$ & 243.0 & 341.7 \\
\hline 30 & HC 5 & 18.2 & 17.3 & 159.0 & 296.0 \\
\hline \multicolumn{2}{|r|}{ General Mean } & 18.2 & 19.0 & 237.2 & 308.3 \\
\hline \multicolumn{2}{|r|}{$\mathrm{CV}(\%)$} & 9.8 & 5.7 & 9.5 & 12.7 \\
\hline \multicolumn{2}{|r|}{$\mathrm{SE}(\mathrm{m})$} & 1.0 & 0.6 & 13.1 & 22.6 \\
\hline \multicolumn{2}{|r|}{$\mathrm{CD}(\mathrm{P} \leq 0.05)$} & 2.9 & 1.8 & 37.0 & 64.1 \\
\hline \multicolumn{2}{|r|}{$\mathrm{CD}(\mathrm{P} \leq 0.01)$} & 3.9 & 2.4 & 49.2 & 85.3 \\
\hline
\end{tabular}

\footnotetext{
$* *$ significant at $1 \%$ and $*$ significant at $5 \%(\mathrm{RF}=$ Rainfed; IR = Irrigated)
}

1. Angle of the primary branch $\left(^{\circ}\right)$

Angle of primary branch is an important trait for mechanical harvesting. Tall genotypes with semi-erect ( 65 to $\left.74^{\circ}\right)$ to erect $\left(75-90^{\circ}\right)$ growth habit are suitable for mechanical harvesting. Angle of primary branch ranged from $69.1^{\circ}$ (JG 11) to $81.1^{\circ}$ (HC 5) under rainfed condition with a general mean value of $74.7^{\circ}$. Under irrigated condition, angle of primary branch ranged from $70.9^{\circ}$ (PG 08108) to $83.2^{\circ}$ (MH 11) with a general mean value of $75.8^{\circ}$. NBeG 780 (rainfed $=80.5^{\circ}$, irrigated $\left.=79.7^{\circ}\right)$, ICCV $181602\left(\right.$ rainfed $=77.5^{\circ}$, irrigated $=$ $78.3^{\circ}$ ), ICCV 181608 (rainfed $=78.8^{\circ}$, irrigated $=79.3^{\circ}$ ), $\mathrm{NBeG} 47$ (rainfed $=78.5^{\circ}$, irrigated $=80.9^{\circ}$ ) and $\mathrm{HC} 5$ $\left(\right.$ rainfed $=81.1^{\circ}$, irrigated $\left.=78.0^{\circ}\right)$ performed significantly superior over the general mean under both rainfed and 
irrigated conditions. Under irrigated condition, ICCV 181606 $\left(78.3^{\circ}\right)$, ICCV $181610\left(78.7^{\circ}\right)$ and $\mathrm{MH} 11\left(83.2^{\circ}\right)$ had significantly superior values over the mean. Under rainfed MH $5\left(77.5^{\circ}\right)$ had erect growth habit with significantly superior values over the general mean. Vishnu et al. (2018) in his study identified ' $\mathrm{NBeG} 1006$ ' and 'NBeG 47' under rainfed condition and 'NBeG 1006', 'NBeG 47', 'NBeG 780' and 'NBeG 855' under irrigated condition as better genotypes for mechanical harvest with semi erect growth habit and desirable angle of the primary branch.

\section{Height of the first pod $(\mathrm{cm})$}

For efficient mechanical harvesting, height of the first pod of chickpea genotypes should be greater than $25-30 \mathrm{~cm}$ from the ground level. The general mean under rainfed condition was $25.3 \mathrm{~cm}$ ranging from $\mathrm{MH} 14(18.4 \mathrm{~cm})$ to ICCV 181664 $(35.4 \mathrm{~cm})$. NBeG $865(27.7 \mathrm{~cm})$, ICCV $181612(30.7 \mathrm{~cm})$, MH $5(30.1 \mathrm{~cm})$, MH $21(28.7 \mathrm{~cm})$, GBM $2(29.0 \mathrm{~cm})$ and HC $5(28.2 \mathrm{~cm})$ exhibited significantly superior values over the mean under rainfed condition. The general mean under irrigated condition was $26.3 \mathrm{~cm}$ ranging from NBeG 868 $(20.5 \mathrm{~cm})$ to ICCV $181664(34.9 \mathrm{~cm})$. MH $11(28.6 \mathrm{~cm})$ and NBeG $47(32.3 \mathrm{~cm})$ showed significantly higher values over mean under irrigated condition. Under both rainfed and irrigated conditions, ICCV 181602 (rainfed $=28.3 \mathrm{~cm}$, irrigated $=29.7 \mathrm{~cm})$, ICCV 181607 (rainfed $=28.7 \mathrm{~cm}$, irrigated $=29.3 \mathrm{~cm}$ ), ICCV $181608($ rainfed $=30.1 \mathrm{~cm}$, irrigated $=29.3 \mathrm{~cm})$, ICCV $181610($ rainfed $=28.7 \mathrm{~cm}$, irrigated $=29.2 \mathrm{~cm})$, ICCV 181664 (rainfed $=35.4 \mathrm{~cm}$, irrigated $=34.9 \mathrm{~cm})$ and ICCV 181667 (rainfed $=27.8 \mathrm{~cm}$, irrigated $=32.5 \mathrm{~cm}$ ) showed significantly better values compared to other genotypes.

\section{Days to $50 \%$ flowering}

Early phenology (early flowering, early podding and early maturity) is the most important mechanism to escape terminal drought stress (Gaur et al., 2008) ${ }^{[6]}$. Chickpea breeding at ICRISAT and in Indian national programme has placed high emphasis on the development of early maturing varieties for enhancing adaptation of chickpea to environments prone to predictable terminal drought stress. In this study, under rainfed conditions, days to $50 \%$ flowering ranged from 34.3 (NBeG 776) to 58.0 (GBM 2) with a general mean of 46.0 whereas under irrigated conditions it ranged from 34.3 (JG 11) to 58.7 (HC 5 and GBM 2) with a general mean of 46.7 . NBeG 776 (rainfed = 34.3, irrigated = 35.3), NBeG 779 $($ rainfed $=35.3$, irrigated $=35.3), \mathrm{NBeG} 780($ rainfed $=36.3$, irrigated $=36.7), \mathrm{NBeG} 868($ rainfed $=36.3$, irrigated $=37.3)$, ICCV 181606 (rainfed = 40.7, irrigated $=41.0$ ), NBeG 47 $($ rainfed $=40.3$, irrigated $=40.7), \mathrm{NBeG} 49($ rainfed $=35.0$, irrigated $=35.7$ ) and JG 11 (rainfed $=35.3$, irrigated $=34.3$ ) showed significant superiority in earliness under both rainfed and irrigated conditions. Some of the genotypes i.e., MH 12 (40.7) under rainfed condition whereas MH 14 (40.3) and MH 15 (42.0) under irrigated condition were significantly early compared to their respective means.

\section{Days to maturity}

Drought escape through early flowering and early maturity are the two major components of drought tolerance for chickpea (Silim and Saxena, 1993) [16]. Under rainfed condition general mean was 89.4 ranging from 81.0 (NBeG 776) to 96.7 (ICCV 181602 and ICCV 181664). The general mean under irrigated condition was 90.1 ranging from 81.7 (NBeG 779) to 98.7 (NBeG 865). Under both rainfed and irrigated conditions, $\mathrm{NBeG} 776$ (rainfed $=81.0$, irrigated $=$ 82.3), NBeG 779 (rainfed $=81.3$, irrigated $=81.7$ ), NBeG 780 $($ rainfed $=82.0$, irrigated $=83.3), \mathrm{NBeG} 868$ (rainfed $=82.3$, irrigated $=84.0)$, ICCV 181610 (rainfed $=86.7$, irrigated $=$ 87.3), ICCV 181612 (rainfed $=86.0$, irrigated $=87.0)$, MH 11 $($ rainfed $=85.3$, irrigated $=87.7), \mathrm{NBeG} 47($ rainfed $=85.3$, irrigated $=88.7), \mathrm{NBeG} 49($ rainfed $=83.7$, irrigated $=84.3)$ and JG 11 (rainfed $=81.3$, irrigated $=82.3$ ) showed significant earliness in maturity over their respective mean values. Genotypes ICCV 181606 (88.0), ICCV 181607 (86.0) and MH 15 (86.3) exhibited significantly superior values over the mean exclusively under irrigated condition. This indicates that in the present material there is opportunity to choose genotypes for desired earliness. Several early maturing high-yielding cultivars have been developed in chickpea. According to a survey conducted on the extent of adoption of chickpea varieties, the short-duration chickpea variety JG 11 covered about $82 \%$ of the chickpea area in Andhra Pradesh (Bantilan et al., 2014) ${ }^{[2]}$.

\section{Duration of reproductive phase}

Duration of reproductive period is also important criterion for realizing higher yields. It is reported that in chickpea, 50-55 days reproduction period is optimum for good seed setting (Joshi et al., 2018) ${ }^{[8]}$. Therefore, early flowering coupled with optimum duration of reproductive period is important for increasing higher yields. Highest reproductive phase was observed in MH 21 (58.0) and the lowest in ICCV 181602 (44.0) under rainfed condition with a general mean of 50.7 and genotypes such as MH 13 (55.0) and MH 21 (58.0) had significantly higher values over mean under rainfed condition. Under irrigated condition, general mean is 51.0 with a range of 44.3 (MH 15) to 58.3 (ICCV 08102) and genotypes ICCV 08102 (58.3), ICCV 181608 (56.0), ICCV 181610 (55.0) and MH 22 (55.3) had significantly higher values over mean under irrigated condition. Under both rainfed and irrigated conditions, ICCV 181606 (rainfed $=54.3$, irrigated $=56.3$ ) had significantly higher values over mean.

\section{Plant height (cm)}

In Southern India, chickpea varieties under cultivation attain a plant height of $35-45 \mathrm{~cm}$ during crop period. However, chickpea cultivars with 30 to 40 per cent more height than the existing cultivars are required to make them suitable for mechanical harvesting. Under rainfed condition the plant height in the genotypes of present study ranged from $33.3 \mathrm{~cm}$ (NBeG 868) to $49.5 \mathrm{~cm}$ (ICCV 181664) with a general mean of $39.0 \mathrm{~cm}$. NBeG $780(41.6 \mathrm{~cm})$, ICCV $181612(41.0 \mathrm{~cm})$, ICCV $181667(45.1 \mathrm{~cm})$ and GBM $2(42.0 \mathrm{~cm})$ performed significantly well exclusively under rainfed condition. Under irrigated condition, plant height ranged from $30.9 \mathrm{~cm}$ (JG 11) to $50.3 \mathrm{~cm}$ (ICCV 181664) with a general mean of $40.2 \mathrm{~cm}$ where MH $11(43.7 \mathrm{~cm})$ is the only genotype which had significantly higher value under irrigated condition. ICCV 181602 (rainfed $=43.0 \mathrm{~cm}$, irrigated $=45.7 \mathrm{~cm}$ ), ICCV 181607 (rainfed $=43.1 \mathrm{~cm}$, irrigated $=44.0 \mathrm{~cm}$ ), ICCV 181608 (rainfed $=44.8 \mathrm{~cm}$, irrigated $=45.7 \mathrm{~cm}$ ), ICCV 181610 (rainfed $=43.3 \mathrm{~cm}$, irrigated $=45.8 \mathrm{~cm}$ ), ICCV 181664 (rainfed $=49.5 \mathrm{~cm}$, irrigated $=50.3 \mathrm{~cm}$ ) and NBeG 47 (rainfed $=42.3 \mathrm{~cm}$, irrigated $=46.0 \mathrm{~cm}$ ) are promising under both under both rainfed and irrigated conditions, and attained significantly greater plant height compared to respective mean values. 
7. Number of branches per plant (primary and secondary)

The general mean of primary branches under rainfed condition was 2.5 where the least value was recorded in NBeG 779 (1.4) and the highest value was recorded in NBeG 865 (3.4). Under irrigated condition, the general mean of primary branches was 2.7 where the least value was recorded in NBeG 47 (1.8) and NBeG 865 (3.3), ICCV 181664 (3.3) and ICCV 181667 (3.3) genotypes recorded extreme values under irrigated conditions. NBeG 865 (rainfed $=3.4$, irrigated $=3.3$ ), ICCV 181664 (rainfed = 3.3, irrigated = 3.3) and ICCV 181667 (rainfed $=3.1$, irrigated $=3.3$ ) performed significantly superior under both rainfed and irrigated conditions. Under rainfed condition, ICCV 181602 (3.1), ICCV 181607 (3.0), MH 4 (3.3) and MH 22 (3.1) also had significant superior values.

The general mean of secondary branches was 7.8 and 8.0 respectively under rainfed and irrigated conditions. The range was $4.2(\mathrm{MH} 12)$ to 13.4 (NBeG 868) under rainfed and 6.5 (ICCV 08102) to 11.9 (NBeG 49) under irrigated condition. Under rainfed condition, $\mathrm{NBeG} 776$ (11.0) and $\mathrm{NBeG} 780$ (10.9) had significantly superior values compared to the mean. $\mathrm{NBeG} 868$ (rainfed $=13.4$, irrigated $=9.5)$, NBeG 49 $($ rainfed $=12.9$, irrigated $=11.9)$ and JG $11($ rainfed $=11.9$, irrigated $=9.9$ ) performed significantly better under both the conditions.

\section{Number of pods per plant}

The general mean value under irrigated condition was greater compared to rainfed (rainfed $=27.6$, irrigated $=31.6$ ). Under rainfed condition, the number of pods per plant ranged from 16.0 (MH 12) to 38.9 (MH 14). ICCV 181606 (37.1), ICCV 181607 (38.5), ICCV 181608 (34.2), MH 4 (33.4), MH 13 (35.4), MH 14 (38.9), NBeG 49 (31.7) and JG 11 (34.3) had significantly superior values. Under irrigated condition the range observed was from 17.9 (ICCV 181602) to 47.9 (MH 15) and two genotypes MH 22 (42.5) and MH 15 (47.9) had significantly higher number of pods per plant compared to mean value.

\section{Biological yield (g/plot)}

Under rainfed condition, biological yield ranged from $388.3 \mathrm{~g}$ (NBeG 47) to $820 \mathrm{~g}$ (MH 13) with a mean value of $543.2 \mathrm{~g}$. Under irrigated condition, biological yield ranged from 490.0 $\mathrm{g}(\mathrm{NBeG} 47)$ to $850.0 \mathrm{~g}$ (MH 4) with a mean value of $654.0 \mathrm{~g}$. Under rainfed, genotypes MH 1 (736.7 g) and MH 14 (740.0 g) exhibited significantly superior values over its mean. Under irrigated condition, NBeG 865 (776.7 g), NBeG 868 $(756.7 \mathrm{~g}), \mathrm{MH} 4(850.0 \mathrm{~g})$ and $\mathrm{MH} 15$ (793.3 g) exhibited significantly superior values over its mean. MH 13 (rainfed = $820.0 \mathrm{~g}$, irrigated $=790.0 \mathrm{~g}$ ) had higher biological yield under rainfed as well as under irrigated conditions.

\section{Harvest Index (\%)}

Improved harvest index represents increased physiological capacity to mobilize photosynthates and translocate them into organs having economic yield. Genotypes with high harvest index are of greater promise for improving drought tolerance in chickpea. Under rainfed condition $\mathrm{HI}$ ranged from 26.1 per cent (MH 5) to 59.8 per cent (JG 11) with a general mean of 43.6 per cent. NBeG 780 (52.5 per cent), ICCV 181606 (56.0 per cent) and ICCV 181607 (51.7 per cent) showed significantly higher values over mean value under rainfed condition. Harvest index ranged from 26.9 per cent (ICCV $181664)$ to 56.4 per cent (NBeG 868) with a mean value of 46.8 per cent under irrigated condition. ICCV 08102 (51.7 per cent), MH 12 (52.1 per cent), MH 22 (52.4 per cent), NBeG 47 (54.0 per cent) and GBM 2 (54.2 per cent) showed significantly higher values over mean value under irrigated conditions. Six genotypes i.e., NBeG 776 (rainfed = 59.2 per cent, irrigated $=53.9$ per cent $), \mathrm{NBeG} 779($ rainfed $=54.9$ per cent, irrigated $=54.6$ per cent $),$ NBeG 868 (rainfed $=59.0$ per cent, irrigated $=56.4$ per cent), $\mathrm{MH} 14$ (rainfed $=54.6$ per cent, irrigated $=55.6$ per cent $), \mathrm{NBeG} 49$ (rainfed $=59.6$ per cent, irrigated $=53.3$ per cent) and JG 11 (rainfed $=59.8$ per cent, irrigated $=53.6$ per cent) showed significantly higher values over their respective means both under rainfed and irrigated conditions.

\section{100 seed weight $(\mathrm{g})$}

The lowest 100 seed weight was observed in MH 5 (rainfed = $16.0 \mathrm{~g}$, irrigated $=17.7 \mathrm{~g}$ ) and the highest value was recorded by $\mathrm{NBeG} 865$ (rainfed $=32.3 \mathrm{~g}$, irrigated $=33.7 \mathrm{~g}$ ). NBeG 776 (rainfed $=29.3 \mathrm{~g}$, irrigated $=28.3 \mathrm{~g}), \mathrm{NBeG} 779$ (rainfed $=29.7 \mathrm{~g}$, irrigated $=29.3 \mathrm{~g}), \mathrm{NBeG} 780($ rainfed $=31.7 \mathrm{~g}$, irrigated $=31.0 \mathrm{~g}$ ), $\mathrm{NBeG} 865$ (rainfed $=32.3 \mathrm{~g}$, irrigated $=$ $33.7 \mathrm{~g}$ ), ICCV 181602 (rainfed $=30.7 \mathrm{~g}$, irrigated $=31.7 \mathrm{~g}$ ), ICCV 181607 (rainfed $=32.0 \mathrm{~g}$, irrigated $=32.7 \mathrm{~g}$ ), ICCV 181664 (rainfed $=27.3 \mathrm{~g}$, irrigated $=27.7 \mathrm{~g}$ ), MH 11 (rainfed $=26.7 \mathrm{~g}$, irrigated $=29.0 \mathrm{~g}), \mathrm{NBeG} 47$ (rainfed $=31.0 \mathrm{~g}$, irrigated $=29.0 \mathrm{~g}$ ) and $\mathrm{NBeG} 49$ (rainfed $=31.3 \mathrm{~g}$, irrigated $=$ $31.0 \mathrm{~g}$ ) had significantly greater 100 seed weight over their respective mean values (rainfed $=24.9 \mathrm{~g}$, irrigated $=25.4 \mathrm{~g}$ ) under both the conditions. Seed weight appears to be more stable across seasons and environments with less $\mathrm{G} \times \mathrm{E}$ interaction (Serraj et al., 2004) ${ }^{[15]}$. MH 14 (28.0 g) and JG 11 $(27.0 \mathrm{~g})$ had significantly greater 100 seed weight under rainfed and irrigated conditions respectively.

\section{Protein content $(\%)$}

Under rainfed conditions protein percent ranged from $14.7 \%$ (MH 21) to $22.2 \%$ (ICCV 181612). ICCV 181612 (22.2\%) was significantly superior to the mean under rainfed. Under irrigated conditions protein percent ranged from $14.5 \%(\mathrm{MH}$ 12 ) to $24.5 \%$ (ICCV 181608). NBeG 865 (24.2\%), ICCV 08102 (21.5\%), ICCV 181608 (24.5\%), MH 1 (21.4\%), MH 4 (22.4\%), MH $11(23.5 \%)$ and GBM 2 (24.2\%) had significantly superior values over the mean exclusively under irrigated condition.

\section{Seed yield (g/plot)}

In Andhra Pradesh more than 80 per cent of chickpea area is under rainfed cultivation and very little rainfall received during crop season. Therefore, the crop is subjected to increased intensity of water deficit which imposes a ceiling on crop duration demanding selection for matching duration varieties for best adaptability and productivity (Saxena 1987 and Ludlow and Muchow, 1990) ${ }^{[14,10]}$. In the present study during current crop season dry weather prevailed in Andhra Pradesh, rainfall was not received for taking up rainfed sowings. Therefore, a pre sowing irrigation was given for sowing rainfed and irrigated crops. Subsequently, the irrigated crop was provided with two light irrigations at 35 and 55 DAS through sprinklers. The mean seed yield of the genotypes was high under irrigated conditions. Though different genotypes have shown differences in their ability to respond to irrigation, the overall improvement in mean performance under irrigated conditions is clearly visible for traits number of pods per plant, $\mathrm{HI}$ and biological yield (Table $3)$. 
Table 3: Significantly superior genotypes based on the mean performance for yield, yield components and mechanical harvestable traits under rainfed and irrigated conditions

\begin{tabular}{|c|c|c|c|c|}
\hline \multirow{2}{*}{$\begin{array}{c}\text { S. } \\
\text { No. }\end{array}$} & \multirow{2}{*}{ Characters } & \multicolumn{2}{|r|}{ Genotypes } & \multirow{2}{*}{ Rainfed and irrigated } \\
\hline & & Rainfed & Irrigated & \\
\hline 1 & $\begin{array}{l}\text { Angle of primary } \\
\text { branch }\end{array}$ & $\begin{array}{c}\text { HC 5, NBeG 780, ICCV 181608, } \\
\text { NBeG 47, MH } 5 \\
\text { and ICCV } 181602 \\
\end{array}$ & \begin{tabular}{|c|} 
MH 11, NBeG 47, NBeG 780, \\
ICCV 181608, ICCV 181610, ICCV 181602 \\
ICCV 181606 and HC 5 \\
\end{tabular} & $\begin{array}{c}\text { NBeG 47, NBeG 780, HC 5, } \\
\text { ICCV } 181608 \text { and } \\
\text { ICCV } 181602 \\
\end{array}$ \\
\hline 2 & Height of the first pod & $\begin{array}{c}\text { ICCV 181664, ICCV 181612, } \\
\text { ICCV 181608, } \\
\text { MH 5, GBM 2, ICCV 181610, } \\
\text { ICCV 181607, } \\
\text { MH 21, ICCV } 181602, \text { HC 5, } \\
\text { ICCV } 181667 \text { and } \\
\text { NBeG } 865\end{array}$ & $\begin{array}{l}\text { ICCV 181664, ICCV 181667, NBeG 47, } \\
\text { ICCV 181602, ICCV } 181607, \text { ICCV } \\
\text { 181608, ICCV } 181610 \text { and MH } 11\end{array}$ & $\begin{array}{c}\text { ICCV 181664, ICCV 181608, } \\
\text { ICCV 181610, ICCV 181602, } \\
\text { ICCV 181607 and ICCV } \\
181667\end{array}$ \\
\hline 3 & $\begin{array}{l}\text { Days to } 50 \% \\
\text { flowering }\end{array}$ & \begin{tabular}{|c|} 
NBeG 776, NBeG 49, JG 11, \\
NBeG 779, NBeG \\
780, NBeG 868, NBeG 47, ICCV \\
181606 and \\
MH 12
\end{tabular} & $\begin{array}{c}\text { JG 11, NBeG 776, NBeG 779, NBeG 49, } \\
\text { NBeG 780, NBeG 868, MH 15, } \\
\text { ICCV 181606, NBeG } 47 \text { and MH } 14\end{array}$ & $\begin{array}{c}\text { JG 11, NBeG 776, NBeG 779, } \\
\text { NBeG 49, NBeG 780, NBeG } \\
\text { 868, NBeG } 47 \text { and } \\
\text { ICCV } 181606\end{array}$ \\
\hline 4 & Days to maturity & $\begin{array}{c}\text { NBeG 776, NBeG 779, JG 11, } \\
\text { NBeG 780, } \\
\text { NBeG 868, NBeG 49, NBeG 47, } \\
\text { MH 11, } \\
\text { ICCV } 181612 \text { and ICCV } 181610\end{array}$ & $\begin{array}{c}\text { NBeG 779, NBeG 776, NBeG 780, NBeG } \\
\text { 868, NBeG 49, MH 15, ICCV 181612, } \\
\text { ICCV 181610, ICCV 181607, JG 11, } \\
\text { MH 11, NBeG } 47 \text { and ICCV } 181606\end{array}$ & $\begin{array}{c}\text { NBeG 776, NBeG 779, JG 11, } \\
\text { NBeG 780, NBeG 868, NBeG } \\
\text { 49, NBeG 47, MH 11, ICCV } \\
181612 \text { and ICCV } 181610\end{array}$ \\
\hline 5 & $\begin{array}{c}\text { Duration of } \\
\text { reproductive phase }\end{array}$ & MH 21, MH 13 and ICCV 181606 & $\begin{array}{c}\text { ICCV 08102, ICCV 181606, ICCV 181608, } \\
\text { MH } 22 \text { and ICCV } 181610\end{array}$ & ICCV 181606 \\
\hline 6 & Plant height & \begin{tabular}{|} 
ICCV 181664, ICCV 181667, \\
ICCV 181608, \\
ICCV 181610, ICCV 181607, \\
ICCV 181602, \\
NBeG 47, NBeG 780, GBM 2 and \\
ICCV 181612
\end{tabular} & $\begin{array}{l}\text { ICCV 181664, NBeG 47, ICCV } 181610, \\
\text { ICCV 181602, ICCV 181608, ICCV } 181607 \\
\text { and MH } 11\end{array}$ & $\begin{array}{l}\text { ICCV } 181602, \text { ICCV } 181664, \\
\text { ICCV 181607, ICCV } 181608, \\
\text { ICCV } 181610 \text { and NBeG } 47\end{array}$ \\
\hline 7 & $\begin{array}{l}\text { Number of primary } \\
\text { branches } \\
\text { plant }^{-1}\end{array}$ & \begin{tabular}{|c|} 
NBeG 865, ICCV 181664, ICCV \\
181612, MH 4, \\
ICCV 181667, ICCV 181602, MH \\
22 and \\
ICCV 181607 \\
\end{tabular} & NBeG 865, ICCV 181664 and ICCV 181667 & $\begin{array}{c}\text { NBeG 865, ICCV } 181664 \text { and } \\
\text { ICCV } 181667\end{array}$ \\
\hline 8 & $\begin{array}{c}\text { Number of secondary } \\
\text { branches } \\
\text { plant }^{-1}\end{array}$ & $\begin{array}{c}\text { NBeG 868, NBeG 49, JG 11, NBe } \\
\text { G } 776 \text { and } \\
\text { NBeG } 780\end{array}$ & NBeG 49, JG 11 and NBeG 868 & $\begin{array}{l}\text { NBeG } 868, \text { NBeG } 49 \text { and } \\
\text { JG } 11\end{array}$ \\
\hline 9 & $\begin{array}{l}\text { Number of pods } \\
\text { plant }^{-1}\end{array}$ & $\begin{array}{c}\text { MH 14, ICCV 181607, ICCV } \\
\text { 181606, MH 13, } \\
\text { JG 11, ICCV 181608, MH } 4 \text { and } \\
\text { NBeG 49 }\end{array}$ & MH 15 and MH 22 & - \\
\hline 10 & Biological yield plot ${ }^{-1}$ & MH 13, MH 14 and MH 1 & $\begin{array}{c}\text { MH 4, MH } 15, \text { MH } 13, \text { NBeG } 865 \text { and } \\
\text { NBeG } 868\end{array}$ & MH 13 \\
\hline 11 & Harvest index & $\begin{array}{c}\text { JG 11, NBeG 49, NBeG 776, } \\
\text { NBeG 868, ICCV } \\
\text { 181606, NBeG 779, MH 14, } \\
\text { NBeG 780 and } \\
\text { ICCV } 181607\end{array}$ & $\begin{array}{l}\text { NBeG 868, MH 14, NBeG 779, GBM 2, } \\
\text { NBeG 47, NBeG 776, JG 11, NBeG 49, } \\
\text { MH 22, MH } 12 \text { and ICCV } 08102\end{array}$ & $\begin{array}{c}\text { JG 11, NBeG 49, NBeG 776, } \\
\text { NBeG 868, NBeG } 779 \text { and } \\
\text { MH } 14\end{array}$ \\
\hline 12 & 100 seed weight & $\begin{array}{c}\text { NBeG } 865, \text { ICCV } 181607, \mathrm{NBeG} \\
\text { 780, NBeG 49, } \\
\text { NBeG 47, ICCV 181602, NBeG } \\
\text { 779, NBeG 776, } \\
\text { MH 14, ICCV } 181664 \text { and MH } 11\end{array}$ & $\begin{array}{c}\text { NBeG 865, ICCV 181607, NBeG 780, } \\
\text { NBeG 49, NBeG 47, MH 11, ICCV 181602, } \\
\text { NBeG 779, NBeG 776, ICCV } 181664 \text { and } \\
\text { JG11 }\end{array}$ & $\begin{array}{l}\text { NBeG 865, ICCV } 181607, \\
\text { NBeG 780, NBeG 49, NBeG } \\
\text { 47, MH 11, ICCV } 181602, \\
\text { NBeG 779, NBeG } 776 \text { and } \\
\text { ICCV } 181664\end{array}$ \\
\hline 13 & Protein & ICCV 181612 & $\begin{array}{c}\text { ICCV } 181608, \text { NBeG } 865, \text { GBM 2, MH 11, } \\
\text { MH 4, ICCV } 08102 \text { and MH } 1\end{array}$ & 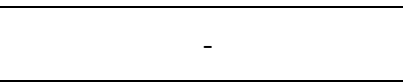 \\
\hline 14 & Seed yield plot $^{-1}$ & $\begin{array}{c}\text { MH 14, NBeG 868, NBeG 49, } \\
\text { NBeG 776, } \\
\text { MH 13, MH 1, NBeG 779, ICCV } \\
181606, \\
\text { ICCV } 181607 \text { and JG } 11\end{array}$ & $\begin{array}{c}\text { NBeG 868, NBeG 779, MH 15, } \\
\text { NBeG 776, MH 4, NBeG } 865 \text { and NBeG } 49\end{array}$ & $\begin{array}{l}\text { NBeG 868, NBeG 776, } \\
\text { NBeG } 779 \text { and NBeG } 49\end{array}$ \\
\hline
\end{tabular}

The general mean of seed yield per plot under rainfed conditions was 237.2 (g) ranging from $139.0 \mathrm{~g}$ (ICCV $181664)$ to $403.3 \mathrm{~g}$ (MH 14). Under irrigated condition seed yield ranged from $141.7 \mathrm{~g}$ (ICCV 181664) to $426.7 \mathrm{~g}$ (NBeG 868) with a general mean of $308.3 \mathrm{~g}$. Seed yields were high under irrigated condition for all the tested genotypes except
NBeG 780, ICCV 181607 and MH 14. Genotypes varied in their ability to respond to irrigation. The increase of seed yield in genotypes under irrigated condition ranged from $1.9 \%$ (MH 13 and ICCV 181664) to 145.4\% (ICCV 08102). 15 genotypes showed more than 20 per cent yield advantage under irrigated condition. Turner et al. (2001), Rao et al. 
(2003) and Meena and Kumar (2015) ${ }^{[17,13,11]}$ also reported that the genotypes of their study responded well to irrigation in terms of yield.

NBeG 776 (rainfed $=318.3 \mathrm{~g}$, irrigated $=383.7 \mathrm{~g}$ ), NBeG 779 $($ rainfed $=305.0 \mathrm{~g}$, irrigated $=391.3 \mathrm{~g}), \mathrm{NBeG} 868$ (rainfed $=$ $361.7 \mathrm{~g}$, irrigated $=426.7 \mathrm{~g}$ ) and $\mathrm{NBeG} 49$ (rainfed $=340.0 \mathrm{~g}$, irrigated $=373.3 \mathrm{~g}$ ) produced significantly superior yield over their respective mean values under both rainfed and irrigated conditions. Genotypes ICCV 181606 (303.3 g), ICCV 181607 (300.0 g), MH 1 (315.0 g), MH 13 (316.7 g), MH 14 (403.3 g) and JG 11 (295.3 g) produced significantly superior yield over the mean under rainfed. NBeG 865 (380.0 g), MH 4 (383.3 g) and MH 15 (386.7 g) produced significantly superior yield over the mean value under irrigated condition.

Among check genotypes $\mathrm{NBeG}$ 47, GBM 2 and HC 5 were tall genotypes suitable for mechanical harvesting released for commercial cultivation in Andhra Pradesh, Karnataka and Hisar respectively. JG 11 is the most popular variety in Andhra Pradesh and NBeG 49 is the recently released high yielding variety for Andhra Pradesh.

Under rainfed condition, among check genotypes NBeG 49 (340.0 g) and JG 11 (295.3 g) were high yielding and MH 14 $(403.3 \mathrm{~g})$ recorded highly significant yield than the checks NBeG 49 and JG 11. NBeG 776, NBeG 779, NBeG 868, ICCV 181606, ICCV 181607, MH 1 and MH 13 were on par with the checks NBeG 49 and JG 11 under rainfed condition. Under irrigated condition, 17 genotypes were on par with the yield of the check NBeG 49 . NBeG 868 recorded significantly high yield than the check JG 11 and nearly 22 genotypes were on par with the yield of the check JG 11.

\section{Conclusion}

In the present study though some types had desirable traits for machine harvesting, many of them are poor yielders. However, under rainfed condition one of the high yielding genotypes, ICCV 181607 had plant height and angle of primary branch comparable with machine harvestable genotype NBeG 47 and the height of first pod was also significantly greater than $\mathrm{NBeG} 47$. Efforts made by earlier researchers to breed for erectness and tall growth habit (Bahl, 1980) ${ }^{[3]}$ have produced tall progenies but with poor harvest index. Plant types with short internodes and compact growth habits have been developed which can be grown under high plant density (Lather, 2000) ${ }^{[9]}$. Therefore, special efforts are needed to breed high yielding genotypes suitable for machine harvesting and with suitability for rainfed cultivation and with favourable response under limited irrigation. Popular and high yielding varieties like $\mathrm{NBeG} 49$ and JG 11, machine harvestable varieties like NBeG 47 and GBM 2 and genotype of present study ICCV 181607 with high yield and as well as machine harvestable traits should be deployed in breeding programmes planned to improve yield and machine harvestablity.

\section{References}

1. AICRP on chickpea Annual report (2017-18). Indian Institute of Pulses Research, Kanpur-208 024.

2. Bantilan MCS, Charyulu DK, Gaur P, Shyam M, Davis J. Short-duration Chickpea Technology: Enabling Legumes Revolution in Andhra Pradesh, India (forthcoming), 2014.

3. Bahl PN. Kabuli-desi introgression and genesis of new plant type in chickpea (Cicer arietinum L.) In: Proceedings of International Workshop on Chickpea
Improvement. ICRISAT, Hyderabad, India, 1980, 7580p.

4. Deshmukh DV, Mhase LB, Jamadagni BM. Evaluation of chickpea genotypes for drought tolerance. Indian $\mathbf{J}$ Pulse Res. 2004; 17:47-49.

5. FAOSTAT. Online Agriculture Statistics, 2019. http..//www.faostat.org.

6. Gaur PM, Krishnamurthy L, Kashiwagi J. Improving drought- avoidance root traits in chickpea (Cicer arietinum L.) - Current status of research at ICRISAT. Plant Prod. Sci. 2008; 11:3-11.

7. Jayalakshmi V, Reddy AT, Kamakshi N, Khayum Ahammad S, Munirathnam P, Gaur PM et al. Prospects of new chickpea varieties in Andhra Pradesh. Proceedings of Inter Drought - V, HICC, Hyderabad, India, 21-25 February, 2017. Poster abstracts, 2017, $187 \mathrm{p}$.

8. Joshi P, Yasin M, Sundaram P. Genetic variability, heritability and genetic advance sudy for seed yield and yield component traits in a chickpea recombinant inbred line (RIL) population. Int. J Pure App. Biosci. 2018; 6(2):136-141.

9. Lather VS. Promising chickpea ideotype for higher plant density. International Chickpea and Pigeonpea Newsletter. 2000; 7:26-27.

10. Ludlow MM, Muchow RC. A critical-evaluation of traits for improving crop yields in water-limited environments. Adv. Agron. 1990; 43:107-153.

11. Meena HP, Kumar J. Estimation of mean performance and genetic association of yield components and drought related traits in chickpea (Cicer arietinum L.). Journal of Legume Research. 2015; 38(1):85-90.

12. Mannur DM. New chickpea variety for mechanical harvesting. Proceedings of Pulses News Letter, Indian Institute of Pulse Research, Kanpur, 2013, 24(1).

13. Rao YK, Durga KK, Reddy MV. Performance of chickpea genotypes under irrigated and unirrigated conditions. International Chickpea Conference, Raipur, India, 2003, 31.

14. Saxena NP. Screening for adaptation to drought: case studies with chickpea and pigeonpea. In: Saxena, N.P., Johansen, C. (eds.) Adaptation of chickpea and pigeonpea to abiotic stresses. Proceedings of Consultant's Workshop, International Crops Research Institute for the Semi-Arid Tropics, Patancheru, India, 1987, 63-76.

15. Serraj R, Krishnamurthy J, Kashiwagi J, Kumar S, Chandran JH, Crouch. Variation in root traits of chickpea (Cicer arietinum. L.) grown under terminal drought. Field Crop Research. 2004; 88:115-127.

16. Silim SN, Saxena MC. Adaptation of spring-sown chickpea to the Mediterranean basis. I. Response to moisture supply. Field Crops Research. 1993; 120:55-62.

17. Turner NC, Wright GC, Siddique KHM. Adaptation of grain legumes (pulses) to water-limited environments to water-limited environments. Advances in Agronomy. 2001; 71:193-231.

18. Vishnu B, Jayalakshmi V, Sudha Rani M. Genetic variability studies on mechanical harvesting traits in chickpea (Cicer arietinum L.). Journal of Food Legumes. 2018; 31(2):75-78. 